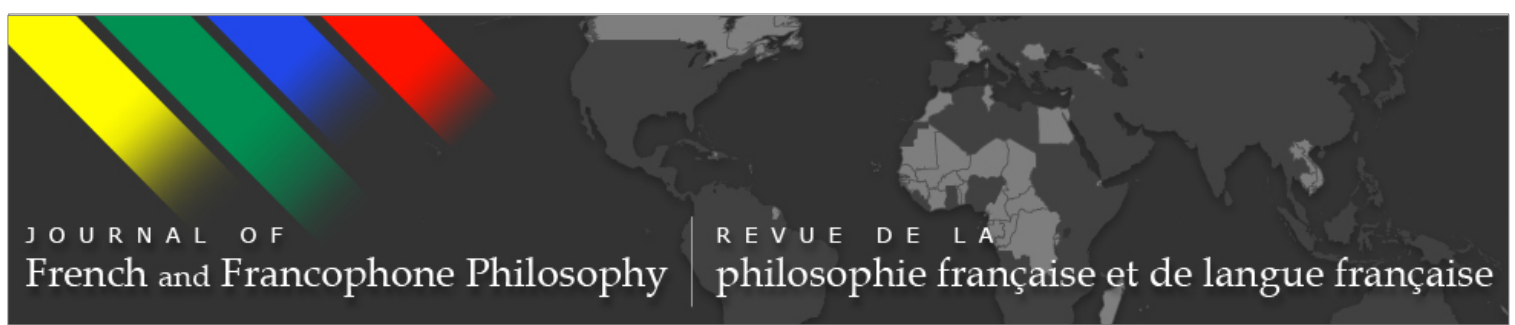

\title{
La France contemporaine face au défi de la créolisation
}

Nathalie Etoke

Journal of French and Francophone Philosophy - Revue de la philosophie française et de langue française, Vol XXV, No 2 (2017) pp. 26-35.

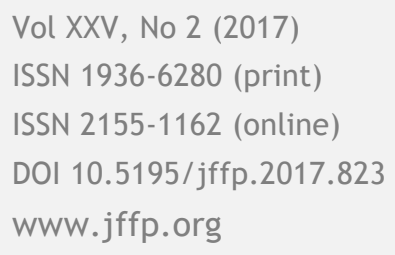

\section{(cc) BY-No-ND}

This work is licensed under a Creative Commons Attribution-Noncommercial-No Derivative Works 3.0 United States License.

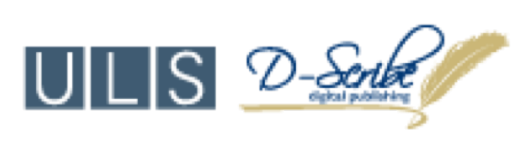

This journal is operated by the University Library System of the University of Pittsburgh as part of its D-Scribe Digital Publishing Program, and is co-sponsored by the University of Pittsburgh Press 


\title{
La France contemporaine face au défi de la créolisation
}

\author{
Nathalie Etoke \\ Connecticut College
}

Creolizing Political Theory: Reading Rousseau through Fanon invite le lecteur à réfléchir à la manière dont les entreprises impérialistes et capitalistes-esclavage transatlantique, colonisation-aboutissent à la création de sociétés, d'identités et d'individus qui remettent en cause les relations de pouvoir et le séparatisme racial inhérents au projet d'origine. Autrement dit, bien que la violence ait rendu possible la ségrégation entre populations étrangères-les européens, les Africains-et les autochtonescaraỉbes, américains natifs-, ce projet initialement séparatiste finit par échouer. Bon gré mal gré, la violence aboutit à des mélanges raciaux et culturels qui, tout en déstabilisant l'autorité conférée à la race blanche, donnent naissance à des croisements involontaires et inévitables. Les frontières établies par la violence et l'imposition de rapports de pouvoir inégaux se révèlent fondamentalement poreuses. Soumises à des transgressions plurielles, ces frontières favorisent l'émergence d'une culture, d'une pensée et d'une manière d'être au monde à la fois locales et singulières. Comme le souligne Jane Anna Gordon, le processus de créolisation: "has referred to distinctive ways in which opposed, unequal groups forged mutually instantiating practices in contexts of radical historical rupture, ones through which people from elsewhere became indigenous to what had recently been foreign places by breaking with the trajectories that their previous collective genealogies would have anticipated." ${ }^{1}$ L'universitaire estime que ce processus de créolisation peut servir de cadre conceptuel. Il permettrait de repenser la manière dont se configurent les sociétés démocratiques contemporaines. Une telle entreprise devient possible à partir d'une méthodologie scientifique qui transcende la catégorisation disciplinaire:

To creolize social scientific and theoretical approaches then is to break with an identity-oriented understanding of disciplines and methods in which one and one's work

Journal of French and Francophone Philosophy | Revue de la philosophie française et de langue française Vol XXV, No 2 (2017) | www.jffp.org | DOI 10.5195/jffp.2017.823 
can only emerge as meaningful by being isomorphic with pre-existing conceptions of what scholarly designation would indicate one must do. If used as an approach to scholarship, its aim is instead guided by another telos: that of contributing to the construction of an inhabitable social world or one in which people can live together as human beings on terms that are neither degrading nor dehumanizing. In so doing, one cannot but grapple with how to think among multiple registers in conversations that do not all partake the same conventions. ${ }^{2}$

D'un point de vue méthodologique mes travaux de recherche pourraient correspondre aux critères énoncés par Gordon. Melancholia africana, l'indispensable développement de la condition noire ${ }^{3}$ et le documentaire Afro Diasporic French Identities ${ }^{4}$ adoptent une démarche transdisciplinaire qui témoigne de mon intérêt pour les productions culturelles africaines et diasporiques. L'analyse d'une œuvre de littérature par exemple, ne se réduit pas à la critique littéraire, elle propose surtout des pistes de réflexion existentialistes sur le rapport entre histoire intime et histoire nationale chez les Africains originaires du continent et ceux de la Diaspora. L'étude des genres musicaux tels que les spirituals, le blues et le jazz met en avant une poétique de l'existence sur la relation à soi, à l'autre ou l'adversité. La critique d'un fait de langue souligne les antinomies de la survie en postcolonie. Le documentaire Afro Diasporic French Identities met en relief les ambivalences du message républicain de liberté, d'égalité et de fraternité vis à vis de l'impensé racial. À travers une série d'interviews d'individus $d^{\prime}$ ascendance africaine et afrodiasporiques issus de milieux divers, j'explore les implications politiques et existentielles de l'être noir dans une société prétendument aveugle à la race. La France demeure prisonnière d'une aporie identitaire fondamentale: son universalisme tant révéré est empreint d'impérialisme. Par conséquent, le projet républicain présenté comme rédempteur d'un passé de domination et d'exploitation n'a toujours pas résolu la situation conflictuelle et particulière dans laquelle se trouvent les descendants d'esclaves et de colonisés. Afro Diasporic French Identities incorpore des voix multiples, elles mêmes entrecoupées de citations de Frantz Fanon, James Baldwin et W.E.B. Du Bois. Des intermèdes musicaux, chorégraphiques ou dramatiques servent de transition entre les différentes parties du documentaire. Que ce soit sous la forme de l'essai ou du documentaire, la question de l'humain et du non humain sert de moteur à la réflexion. Comment être humain dans un monde qui déshumanise? Que signifie l'être noir dans un monde blanc? Dans quelle mesure les héritages post-esclavagistes et post-coloniaux façonnent-ils des manières d'être au monde où la perte et la souffrance se doublent d'une résistance face à la mort, à la folie et au désespoir? Comment le processus de subjectivation 
ancré dans la précarité et l'imprévisibilité accouche-t-il d'une vitalité existentielle qui se déploie dans un monde hostile? Les conséquences sociales, politiques, culturelles et existentielles de la traite négrière, de l'esclavage transatlantique et de la colonisation sont au cœur de ma recherche qui traverse plusieurs espaces géographiques-France, Etats-Unis, Caraibe, Afrique subsaharienne-et linguistiques-le français et l'anglais. Que j'écrive en anglais ou en français, que je parle de l'Afrique continentale ou diasporique, je ne me soucie pas des catégories censées m'inscrire dans un champ spécifique à ma formation universitaire. Afin de résoudre les problèmes qui se posent à nous, il faut d'emblée reconnaître que les disciplines scientifiques sont elles-mêmes enracinées dans l'histoire impérialiste des sociétés qui les ont engendrées et légitimées. De plus, afin de créer un monde habitable pour tous, il convient d'introduire de l'inventivité dans l'existence en établissant de nouvelles grilles d'analyse qui ne reproduisent pas une hiérarchisation de $l^{\prime}$ humain. ${ }^{5}$

Sans pour autant employer le vocable "créolisation", je m'intéresse aux points de tension, de contradiction, de rupture, de continuité et de discontinuité, d'équilibre et de déséquilibre issus du rapport entre colonisateurs et colonisés. Comment se module un monde en devenir qui oblige à repenser l'humain à partir d'une décolonisation de la liberté? Les mythes fondateurs de la démocratie en Occident étant indissociables du projet impérialiste, la liberté a été conçue comme propriété exclusive et caractéristique d'un groupe d'individus particuliers. Or, comme le précise Gordon: "It is in the relation of the colonizer and the colonized and the worlds that erupt between them that we begin to understand what becomes of the human being in the modern world and what, under such circumstances can be done to and with freedom." ${ }^{6}$ Bien que l'universitaire se focalise ici sur le colonisateur et le colonisé dans l'espace colonial, je voudrais reformuler ce constat dans le contexte français hexagonal. En effet, si le devenir des sociétés caribéennes permet d'envisager les possibles de la créolisation, ceux ci s'expriment de manière moins fluide en Europe. La France hexagonale est aujourd'hui le lieu où la relation entre l'ancien colonisateur et l'ancien colonisé crée un monde créolisé. Cependant, ce dernier se heurte à des résistances structurelles, politiques et culturelles. Si pendant des siècles, la France a pris possession de territoires et d'individus sur plusieurs continents, elle fait aujourd'hui l'expérience de la présence sur son sol de populations originaires d'espaces que la République a dominés. Lorsqu'elle est abordée, la question de l'immigration opère comme un trompe l'œil, une espèce de raccourci conceptuel témoignant d'un refus de penser les identités françaises post-coloniales d'ascendance maghrébine, subsaharienne ou caribéenne comme étant nées d'un processus de créolisation spécifique à la France. Ce processus interroge désormais les possibles d'une francité dont les racines se situent au croisement des apories qu'elle a enfantées - les lumières et l'esclavage, la République et la colonie.

Journal of French and Francophone Philosophy | Revue de la philosophie française et de langue française Vol XXV, No 2 (2017) | http://www.jffp.org | DOI 10.5195/jffp.2017.823 
À la question du vivre ensemble est associée celle d'être libres et égaux ensemble. Lorsque les hommes politiques et les intellectuels néoconservateurs parlent de l'échec de l'intégration, ces pourfendeurs d'une certaine idée de la France reconnaissent de manière négative la réalité d'une créolisation sociale à laquelle ils souhaitent mettre fin. ${ }^{7}$ La parole des dominants exprime ainsi une volonté d'établir une hiérarchie entre les valeurs, les cultures et les races. Les crispations actuelles sur la nation et l'identité française révèlent les paradoxes d'une "créolisation [qui] semble ruiner 1 "“autorité" et maintenir le "pouvoir" et la "domination" sans les créoliser." $^{8}$

Les intellectuels les plus en vue du moment: Eric Zemmour, Alain Finkielkraut, Michel Onfray et Pascal Bruckner sont engagés dans des "projects of decreolization [...] in which efforts are made to purify cultures of what are seen as external and contaminating prior or current influences." ${ }^{\prime 9}$ Finkielkraut rapporte les propos d'une personne interviewée par le journal Le monde: "[...] il est pénible de voir des quartiers de Tourcoing ressembler à la " casbah": " [...] Ici dans un quartier d'origine musulmane on n'est plus chez soi." ${ }^{10} \mathrm{Au}$ préalable, le philosophe affirme: "Plus de deux tiers des Français estiment qu'il y a trop d'étrangers en France, et ils sont presqu'aussi nombreux à dire qu'on ne se sent plus chez soi comme avant." ${ }^{11}$ Eric Zemmour estime qu' “à partir des années 1970, lorsque les premiers enfants de l'immigration maghrébine débarquèrent en France, ou naquirent dans l'Hexagone [...]. Le choix du prénom devint un signe politique en tout cas militant." "12 À propos de la visibilité de l'appartenance religieuse, Bruckner déclare: "Le voile, la burqa, le burkini représentent des instruments de conquête de l'espace public, ce sont des tracts qui appellent à la sédition." ${ }^{13}$ Renaud Camus déclare que la France est victime d'un phénomène de "déculturation" et du "grand remplacement" dont l'élément moteur serait "la colonisation" démographique. Il soutient que la surreprésentation démographique de populations d'ascendance maghrébine et subsaharienne change la société française de manière négative. ${ }^{14} \mathrm{Ce}$ florilège de citations souligne la présence indéniable d'une créolisation qui parce qu'elle déstabilise une identité française blanche et judéo chrétienne contribue paradoxalement au renforcement de celle-ci. Et c'est à ce niveau que j'interpelle Gordon. Quelles seraient ses propositions théoriques? L'universitaire écrit:

Rousseau, if in a neutered form, has been canonized within French society-the source of the legitimating language of the very project of the French Republic. [....] In such rhetoric, 1789 is invoked, now conservatively. A more viable and political response would be to call for the creolizing of Rousseau's general will drawing on the ample resources offered by Frantz Fanon. Such an approach would seek out the debates through which 
difference could move from abstract principles to lines of disagreement fostered by unequal reach and provision of the French state. ${ }^{15}$

Dans le contexte français actuel, la créolisation de la société va de pair avec le refus de créoliser la volonté générale. Si l'on conçoit que la créolisation pourrait autoriser d'autres possibles du politique, il convient aussi de souligner qu'elle: "suppose [que] des éléments culturels mis en présence doivent obligatoirement être "équivalents en valeurs" pour que cette créolisation s'effectue réellement. C'est-à-dire que si dans des éléments culturels mis en relation certains sont infériorisés par rapport à d'autres, la créolisation ne se fait pas vraiment. Elle se fait sur un mode bâtard et sur un mode injuste." ${ }^{16}$ Il ne s'agit plus de nier la différence mais de présupposer une égalité de principe. Aujourd'hui, la France est soumise à une créolisation bâtarde ayant pour corollaire la montée du racisme, de l'inégalité et de l'injustice. Comment penser la créolisation politique dans une ancienne puissance colonisatrice? L'alliance objective des intérêts particuliers d'une France athée, judéo chrétienne, blanche et islamophobe rend possible une transmutation du populisme en volonté générale. C'est ainsi qu'en 2010 le parlement français a voté une loi anti-burqa prohibant le port du voile intégral dans l'espace public. Six années plus tôt, une loi plus générale a interdit le port de signes ou de tenues qui expriment de manière ostensible une appartenance religieuse. Ces deux lois sont des exemples de “commandements [qui] n'apparaissent pas comme l'expression du caprice ou de la volonté arbitraire des autorités civiles, mais comme l'expression de la volonté générale." ${ }^{17}$ En effet, la notion de bien commun est constamment mise en avant pour renforcer des lois anti-musulmanes à la fois punitives et disciplinaires. De plus, parce que ses lois ciblent essentiellement les femmes musulmanes, il se développe en parallèle un discours qui confond volonté générale, identité française et libération obligatoire de la femme musulmane. À titre d'exemple on peut citer la tribune de Manuel Valls intitulée: "En France, les femmes sont libres." ${ }^{18}$ Dans un tel contexte, au lieu de négocier avec les différences, la volonté générale se fait l'écho d'une impulsion totalitaire.

La crispation autour du vêtement religieux est un exemple parmi tant d'autres. On pourrait également évoquer la thématique de la repentance qui empêche d'examiner ce que le passé esclavagiste et colonial a produit d'imprévisible, d'inédit, de complexe et d'aporétique. Nicolas Sarkozy alors président de la République déclare détester:

cette mode de la repentance qui exprime la détestation de la France et de son Histoire. [...] Je déteste la repentance qui dresse les Français les uns contre les autres en fonction de leurs origines. Je déteste la repentance qui est un obstacle à l'intégration parce que l'on a rarement envie de 
s'intégrer à ce que l'on a appris à détester, alors que l'on devrait le respecter et l'aimer. Voilà ma vérité. ${ }^{19}$

La position de l'ancien chef de l'Etat n'est pas exceptionnelle. Nombreux sont les intellectuels qui dénoncent la critique du passé esclavagiste et colonialiste de la France. ${ }^{20}$ Selon Benjamin Stora, on assiste à "une guerre des mémoires." ${ }^{21}$ Qu'il s'agisse de la mémoire ou de la visibilité des signes religieux musulmans, la France est aux prises avec un processus de créolisation irréversible dont la caractéristique essentielle est le "croisement subversif des cultures." ${ }^{22}$ Lorsque les minorités s'organisent pour réclamer des droits, l'intelligentsia médiatico-politique les accuse de communautarisme. Leurs revendications seraient contraires à la volonté générale: "Mais les minorités veulent-elles se fondre dans un ensemble qui les dépasse ou rester distinctes, faire sécession du reste de la société? Ne risquent-elles pas, chacune de dégénérer en micronationalismes, réclamant un traitement spécial, selon la foi, les origines, la couleur de la peau?"23 La tension entre la volonté générale et le droit des minorités témoigne du décalage entre la créolisation de la société et le refus de créoliser les modes de la pensée politique. Or, seule une pensée politique créolisée autorise de nouveaux champs de référence et d'action où la différence et le dissensus ne constituent plus des obstacles mais des opportunités productives orientées vers la recherche du bien commun. ${ }^{24}$ Le refus de créoliser la pensée politique, peut-il seul rendre compte de l'impasse dans laquelle se trouve la France aujourd'hui?

Dans la Caraibe, les populations autochtones ont été décimées ou contraintes de se mêler aux nouveaux arrivants. En dépit d'un système stratifié par la race et le statut de personne libre/non libre, les multiples transgressions et les mélanges ont contribué à éradiquer, fragiliser ou déstabiliser la question des origines et l'idée de pureté ethno-raciale. Par conséquent, la Nation, la Culture et l'Identité deviennent des notions fondamentalement poreuses, tributaires d'un processus de déterritorialisation ${ }^{25}$ et de reterritorialisation. Contrairement à la Caraibe où la créolisation autorise: "this combination of closure and openness, of sedimentation and fluidity, and of particular forms of identification gaining coherence through their recontextualization," ${ }^{26}$ dans l'espace hexagonal, le déplacement de la périphérie vers le centre se produit en termes d'ouverture conditionnée par une structure étatique centralisée et verticale. Autrement dit, il y a la personne qui arrive-l'immigré-et l'autochtone, l'accueilli et l'accueillant. Le nouvel arrivant n'a rien à offrir. Il doit se fondre dans un moule préexistant représentatif d'une homogénéité culturelle et politique plus ou moins cohésive. Ce qui permet à Pascal Bruckner d'affirmer que "quiconque voudrait gommer l'histoire et la personnalité des pays européens, leur imposer une loi étrangère, leur demander de collaborer à leur propre décomposition ou transformer les autochtones en exilés de l'intérieur, se trompe [...] de siècle." ${ }^{27}$ Présente in absentia, la race solidifie 
cette catégorisation entre français de souche et français d'origine immigrée. Le français non blanc-maghrébin, africain subsaharien, asiatique-, peu importe qu'il soit de la deuxième ou de la troisième génération, porte sur son corps la marque de sa non appartenance. Parce que l'on examine sa citoyenneté sous l'angle de la race supposée renvoyer à un territoire non européen et à des systèmes de valeur perçus comme "différents", il ne sera jamais considéré comme étant un français à part entière. Bien qu'ils créolisent la République, ces français maghrébins, subsahariens ou asiatiques ne sont acceptables/acceptés, qu'à partir du moment où s'accomplit à travers leur parcours la prophétie auto-réalisatrice d'une Nation qui réussit à intégrer et à assimiler ses immigrés. Lorsque cette prophétie n'a pas lieu, le fantasme de l'extraction du corps étranger réapparaît. D’où l'idée de la déchéance de nationalité évoquée par le président François Hollande au lendemain des attentats du 13 novembre 2015. Bien que cette initiative populiste ait échoué avec fracas, il convient de préciser que son objectif était d'expulser les bi-nationaux terroristes vers leur prétendu pays d'origine. Dans cette optique, "le retour à une identité homogène imaginaire par la soustraction du corps de la nation des éléments perçus comme hétérogènes" ${ }^{28}$ aurait dû garantir la sécurité et le bien commun. Parce que l'identité est figée dans le temps et l'espace, même s'il y a une rencontre et un brassage de populations, ceux-ci se situent en contrepoint d'un "dilemme républicain à la française": "Il s'agit d'un conflit de valeurs et d'un paradoxe sociologique. On affirme dans l'abstrait et sur le registre de l'explicite des valeurs universelles [...] tous les hommes sont égaux en dignité, en aptitudes, en droits, tandis qu'aux colonies on assiste à des pratiques de hiérarchisation de groupes légitimant des modes de domination et d'exploitation." ${ }^{29}$ Pourtant comme le souligne Jane Gordon:

Freedom requires people continuing to enact their collective sovereign power, actively associating, negotiating, and potentially reconciling their shared and disparate needs in a general will. If this is not genuinely general-if it is instead a particular will of a factious group presenting its concerns and aims as of more abiding-the "political machine" will begin to reinstate the cyclical life of the "right" of the strongest. ${ }^{30}$

Lorsque l'on examine les tensions actuelles au sein de la société française, la manière dont se configurent la souveraineté collective et la volonté générale reflète une opposition continue entre processus de créolisation de la société-au niveau culturel, religieux et ethnique-et anti-créolisation du politique-vote de lois anti-musulmanes, montée du vote ethno-nationaliste blanc. Cette conflictualité génère un repli sur soi qui détourne la volonté générale de sa mission de représentativité du bien commun. Pascal Bruckner écrit: "La France contemporaine ne rêve plus d'impérialisme. Elle vit sur un

Journal of French and Francophone Philosophy | Revue de la philosophie française et de langue française Vol XXV, No 2 (2017) | http://www.jffp.org | DOI 10.5195/jffp.2017.823 
patriotisme de la rétraction et non d'expansion, elle rêve de fermer ses frontières plutôt que de les distendre à l'infini. Son vrai mot d'ordre aujourd'hui serait plutôt "à bas le monde extérieur." ${ }^{\prime 1}$ Le philosophe emploie ici un vocabulaire qui entretient le mythe d'un retour aux origines blanches et judéo-chrétiennes. Les notions de frontière ou de "monde extérieur" sont ressuscitées afin de nier qu'elles ont été abolies depuis plusieurs siècles. D'un point de vue géopolitique, peut-on parler de "monde extérieur" quand ce monde était naguère nommé: Afrique Equatoriale française, Afrique Occidentale française ou Algérie sous tutelle française? Les Départements et Territoires d'Outre-Mer font-ils partie du "monde extérieur"? La France ne pourra pas expurger la composante créolisante qui provoque des changements internes à la fois involontaires, imprévisibles et irréversibles. La créolisation entendue comme croisement productif des apories oblige à repenser le vivre ensemble en termes d'égalité et de liberté pour l'ensemble des citoyens français.

Cependant, la question ultime qui demeure en suspens est celle d'une créolisation politique: "Creolization is borne of just this doubled moment: of loss and melancholy and simultaneously of possibilities, even necessities for self-creation, of fashioning what is supposed to have been effective because primordial." ${ }^{32}$ Aujourd'hui l'Europe d'une manière générale, la France en particulier voit disparaître une certaine idée de l'Identité et de la Nation. Elle est le théâtre de conflictualités sociales, culturelles, démographiques et politiques. Le processus de créolisation du politique demeure impossible parce qu'au lieu de devenir des catalyseurs de créativité ou de transformation autorisant l'exploration des possibles, la perte et la mélancolie renforcent un repli sur soi doublé de nostalgie. Il ne s'agit pas d'être pour ou contre la créolisation de la société. Elle a déjà eu lieu. Tant que la créolisation sera appréhendée comme un problème à résoudre au lieu d'une opportunité à saisir, pourra-t-on jamais comprendre le monde dans lequel nous vivons et relever les défis politiques qui s'imposent à nous?

1 Jane Anna Gordon, Creolizing Political Theory: Reading Rousseau Through Fanon (New York: Fordham University Press, 2014), 2-3.

2 Jane Anna Gordon, "Creolizing as The Transdisciplinary Alternative to Intellectual Legitimacy on the Model of the "Normal Scientific" Community," quaderna.org, 13 Oct., http: / / quaderna.org/creolizing-as-the-transdisciplinary-alternative-tointellectual-legitimacy-on-the-model-of-the-normal-scientific-community/ 
${ }^{3}$ Nathalie Etoke, Melancholia africana l'indispensable dépassement de la condition noire (Paris: Editions du Cygne, 2010).

${ }^{4}$ Nathalie Etoke, Afro Diasporic French Identities, DVD, 2011.

5 Jane Anna Gordon, "Creolizing as The Transdisciplinary Alternative to Intellectual Legitimacy on the Model of the "Normal Scientific" Community."

6 Jane Anna Gordon, Creolizing Political Theory, 79.

7 Jane Anna Gordon, Creolizing Political Theory, 186.

8 Edelyn Dorismond, “Créolisation de la politique, politique de la créolisation. Penser un “im-pensé” dans l'œuvre d'Edouard Glissant”, Cahiers Sens public (2009):

137-138.

9 Jane Anna Gordon, Creolizing Political Theory, 186.

${ }^{10}$ Alain Finkielkraut, La seule exactitude (Paris: Stock, 2015), 27.

${ }^{11}$ Finkielkraut, La seule exactitude, 26.

${ }^{12}$ Eric Zemmour, Le suicide français, (Paris: Albin Michel, 2014), 371.

13 Pascal Bruckner, Un racisme imaginaire: Islamophobie et culpabilité (Paris: Bernard Grasset, 2017), 78.

14 Renaud Camus, Dernière chance avant le Grand Remplacement: Changer de peuple ou changer de politique? (La Maison d'Edition, 1, 2017).

15 Jane Anna Gordon, Creolizing Political Theory, 159.

${ }^{16}$ Edouard Glissant, Introduction à une poétique du divers (Paris: Gallimard, 1996), 17.

17 Luc Foisneau, “Gouverner selon la volonté générale: la souveraineté selon Rousseau et les théories de la raison d'Etat," Les Études philosophiques, $\mathrm{n}^{\circ} 83$ (2007), 468.

18 Manuel Valls, “En France, les femmes sont libres", huffingtonpost.fr, 13 Oct. 2017: http://www.huffingtonpost.fr/manuel-valls/manuel-valls-interdiction-burkiniislam-laicite_b_11865808.html.

19 Le discours de Nicolas Sarkozy, liberation.fr, 13 Oct. 2017, http://www.liberation.fr/france/2007/05/06/le-discours-de-nicolas-sarkozy_9889 ${ }^{20}$ Alain Griotteray, Je ne demande pas pardon. La France n'est pas coupable (Paris: La Table Ronde, 2006), Paul François Paoli, Nous ne sommes pas coupables assez de repentance (Paris: Éditions du Rocher, 2001), Max Gallo, Fier d'être français (Paris: Fayard, 2006), Pascal Bruckner, La tyrannie de la pénitence: Essai sur le masochisme occidental (Paris: Grasset, 2008).

Journal of French and Francophone Philosophy | Revue de la philosophie française et de langue française Vol XXV, No 2 (2017) | http://www.jffp.org | DOI 10.5195/jffp.2017.823 
21

Benjamin Stora, "La guerre des mémoires, la France face à son passé colonial"

Entretiens avec Thierry Leclère (Paris: Éditions de l'aube, 2011).

${ }^{22}$ Edelyn Dorismond, "Créolisation de la politique, politique de la créolisation.

Penser un “im-pensé” dans l'œuvre d'Edouard Glissant,” 145.

23 Pascal Bruckner, Un racisme imaginaire: Islamophobie et culpabilité, 159

24 Jane Anna Gordon, Creolizing Political Theory, 128.

25 Gilles Deleuze and Felix Guattari, L'Anti-OEdipe: Capitalisme et schizophrénie (Paris: Les Editions de Minuit, 1972).

26 Jane Anna Gordon, Creolizing Political Theory, 169

27 Pascal Bruckner, Un racisme imaginaire: Islamophobie et culpabilité, 219-220

${ }^{28}$ Luc Foisneau, “Gouverner selon la volonté générale," 472.

29 Nicolas Bancel, Pierre-André Taguieff Pierre-André, “Universalisme et racisme évolutionniste: le dilemme républicain”, Hommes et Migrations, $n^{\circ} 1207$, Imaginaire colonial, figures de l'immigré (1997), 91.

30 Jane Anna Gordon, Creolizing Political Theory, 107.

${ }^{31}$ Pascal Bruckner, Un racisme imaginaire: Islamophobie et culpabilité, 189.

32 Jane Anna Gordon, Creolizing Political Theory, 197. 